\title{
Aménagement d'ossuaires dans le baptistère paléochrétien de Brioude (Haute-Loire) : sélection, tri des ossements et relation À l'Âge au décès
}

Ossuary organisation in the paleochristian baptistery at Brioude (Haute-Loire), France: the relationship between age-at-death and skeletal element sorting and selection

Aline Thomas, Fabrice Gauthier, Esther Gatto et Pascal Murail

\section{(2) OpenEdition}

\section{Édition électronique}

URL : https://journals.openedition.org/bmsap/6464

DOI : $10.4000 /$ bmsap.6464

ISSN : 1777-5469

Éditeur

Société d'Anthropologie de Paris

Édition imprimée

Date de publication : 1 juin 2009

ISSN : 0037-8984

Référence électronique

Aline Thomas, Fabrice Gauthier, Esther Gatto et Pascal Murail, « Aménagement d'ossuaires dans le baptistère paléochrétien de Brioude (Haute-Loire) : sélection, tri des ossements et relation À l'Âge au décès », Bulletins et mémoires de la Société d'Anthropologie de Paris [En ligne], 21 (1-2) | 2009, mis en ligne le 07 juin 2010, consulté le 01 juin 2021. URL : http://journals.openedition.org/bmsap/6464 ; DOI : https://doi.org/10.4000/bmsap.6464

\section{(c) $(1) \odot$}

Les contenus des Bulletins et mémoires de la Société d'Anthropologie de Paris sont mis à disposition selon les termes de la licence Creative Commons Attribution-NonCommercial-NoDerivatives 4.0 International License. 


\title{
AMÉNAGEMENT D'OSSUAIRES DANS LE BAPTISTÈRE PALÉOCHRÉTIEN DE BRIOUDE (HAUTE-LOIRE) : SÉLECTION, TRI DES OSSEMENTS ET RELATION À L'ÂGE AU DÉCÈS
}

\author{
OSSUARY ORGANISATION IN THE PALEOCHRISTIAN BAPTISTERY AT BRIOUDE \\ (HAUTE-LOIRE), FRANCE: THE RELATIONSHIP BETWEEN AGE-ET-DEATH \\ AND SKELETAL ELEMENT SORTING AND SELECTION
}

\author{
Aline Thomas ${ }^{1}$, Fabrice Gauthier ${ }^{2}$, Esther Gatto ${ }^{1,2}$, Pascal Murail ${ }^{1}$
}

RÉSUMÉ

Le baptistère de Brioude constitue actuellement un ensemble unique en Auvergne. Intégré au sanctuaire de SaintJulien au début du haut Moyen Âge, il présente des sépultures installées intra muros au moment de son fonctionnement, fait exceptionnel en Gaule mérovingienne.

La datation particulièrement bien établie de l'ensemble des vestiges et, par conséquent, du rythme des changements qu'ils ont subis, a permis d'identifier deux phases funéraires : la première, datée du VI ${ }^{\mathrm{e}} \mathrm{s}$. ap. J.-C., est contemporaine de l'utilisation du baptistère. La seconde, désigne l'ensemble des sépultures installées dans le niveau de démolition du baptistère sur les tombes précédentes ; elles sont associées à un nouvel édifice élevé sur l'emprise du baptistère au début $\mathrm{du} \mathrm{VII}^{\mathrm{e}} \mathrm{s}$.

L'analyse du fonctionnement de l'ensemble de cet espace funéraire a permis d'identifier une étape charnière entre ces deux principales phases chronologiques. Celle-ci correspond au réaménagement de plusieurs sépultures appartenant à la première phase, en raison de la construction de l'édifice funéraire remplaçant le baptistère. Ces perturbations ont entraîné le déplacement ou la destruction partielle de certains sarcophages. Une partie des ossements ainsi exhumés a été rassemblée en ossuaires.

Trois ossuaires ont été mis au jour dans une fosse creusée dans le sol de la salle baptismale. La présence de liaisons ostéologiques de deuxième ordre entre ces différents ensembles démontre, d'une part, que leur mise en place résulte d'un processus unique, d'autre part, que la distribution de l'ensemble des vestiges osseux n'a pas été aléatoire. En effet, une sélection et un tri d'os matures en fonction de leur catégorie (os longs, blocs cranio-faciaux et os de petite et moyenne tailles) ont été réalisés et répartis en trois dépôts distincts. Nous constatons en outre, que les os pairs non matures ont été répartis dans deux ossuaires. Enfin, l'estimation de l'âge de ces jeunes sujets les place majoritairement parmi la classe des 5-9 ans, individus d'ailleurs absents du reste de l'espace sépulcral. Ces résultats laissent envisager l'existence d'une zone originelle réservée à cette classe d'âge au décès de la population, détruite lors de la construction du bâtiment de la seconde phase.

Mots-clés : ossuaire, tri des ossements, baptistère, Moyen Âge, pratiques funéraires.

1. UMR 5199, PACEA, Laboratoire d'Anthropologie des Populations du Passé, Université Bordeaux 1, avenue des Facultés, 33405 Talence CEDEX, France, e-mail : a.thomas@anthropologie.u-bordeaux1.fr

2. Institut National de Recherche Archéologique Préventive, Auvergne, 13bis rue Pierre Boulanger, Le Brézet, 63100 Clermont Ferrand. 


\section{ABSTRACT}

The baptistery of Brioude is today a unique monument in Auvergne. It was associated with the sanctuary housing the relics of Saint Julien, which dates to the early Middle Ages. During its period of use, the baptistery provided for intra muros burial. This is a unique occurrence in Merovingian Gaul.

Two phases of funerary use could be identified from the regularity with which the remains had been treated. The first, dating from the 6th century $A D$, is contemporary with the use of the baptistery. The second dates from the middle of Merovingian period (from the 7th century A.D.) and is associated with the building of a new structure on the site of the baptistery. This structure was accompanied by burials placed directly over those of the previous phase.

The analysis of spatial relationships among burials enabled us to identify a transitional stage between these two phases. This stage was identified on the basis of the disturbance of several burials belonging to the first phase, subsequent to the construction of the structure of the second phase. These disturbances led to the displacement of partial destruction of some sarcophagi and the inclusion of some disturbed skeletal remains in ossuaries.

Three ossuaries have been discovered under the floor of the baptistery. The identification of osteological relations of the second order among these different groups demonstrates that, first, the three ossuaries resulted from a single action and, second, the distribution of skeletal elements was not random but that selection and sorting of adult bones had been carried out according to their anatomical type (long bones, cranium, small and middle-sized bones), and these had been separated into threee different deposits. Furthermore, we noted that paired immature skeletal elements were divided between two ossuaries. Finally, the age assessment of these young individuals classified them into the 5-9 age category, a group that was absent from the rest of the burial area. These results lead us to hypothesize that there had once been a burial area reserved for this part of the population. This area seems to have been destroyed during the construction of the second phase structure.

Keywords: ossuary, bone sorting and selection, baptistery, Middle Ages, burial practices.

\section{INTRODUCTION}

L'histoire de Brioude (Haute-Loire) est intimement liée au culte rendu aux reliques de saint Julien. Cette dévotion, dont témoigne aujourd'hui la célèbre basilique romane Saint-Julien, est en fait attestée dès la deuxième moitié du $\mathrm{V}^{\mathrm{e}}$ s., date à laquelle le vicus accueille la tombe du martyr saint Julien. Les fouilles, successivement menées par G. Fournier puis F. Gauthier entre 1972 et 2006 dans le secteur de l'actuelle basilique, ont conduit à la mise au jour d'une partie des édifices religieux primitifs, notamment du baptistère paléochrétien. Ces découvertes ont parallèlement mis en évidence une concentration d'inhumations ad sanctos, attirées par les reliques du saint.

L'originalité du baptistère de Brioude tient à la présence de tombes au sein même de l'édifice, dont la contemporanéité avec son occupation est attestée grâce au remarquable mobilier (deniers, épitaphes) découvert en contexte stratigraphique (Gauthier 2004; Dhénin, Gauthier 2005).

La datation particulièrement bien établie de l'ensemble des vestiges et, par conséquent, du rythme des changements qu'ils ont subi, a permis d'identifier deux phases funéraires. La première, datée du $\mathrm{VI}^{\mathrm{e}} \mathrm{s}$. ap. J.-C. par le mobilier, correspond à l'ensemble des sarcophages contemporain à l'utilisation du baptistère, découvert à l'intérieur de l'édifice (dans la salle baptismale elle-même et dans son annexe sud) ou à proximité immédiate (contre son mur est) (fig. 1). La seconde phase, postérieure au baptistère, est datée à partir de la deuxième moitié de l'époque mérovingienne ${ }^{3}$. Cette phase funéraire fonctionne avec un nouvel édifice élevé sur l'emprise du baptistère : le bâtiment 5 (fig. 1). Notons qu'un rapport est ostensiblement entretenu entre ce vaste bâtiment (environ $180 \mathrm{~m}^{2}$ ) et le baptistère sur lequel il est implanté. En effet, ces transformations architecturales opérées pendant l'époque mérovingienne illustrent une nette rupture dans le fonctionnement globale du sanctuaire.

3. Des analyses radiocarbones réalisées sur deux tombes associées à ce bâtiment, indiquent une datation commune au milieu du vII ${ }^{\mathrm{e}} \mathrm{s}$. (SP 5102 Code labo: Lyon-13668, Âge $C^{14}$ BP : -1425 +/-30, Âge calibré : de 578 à 658 ap. J.-C., date la plus probable : 641 . SP 5065 Code labo : Lyon-3549 (GrA), Âge C ${ }^{14}$ BP : -1380 +/-30, Âge calibré : de 612 à 670 ap. J.-C., date la plus probable : 641). 


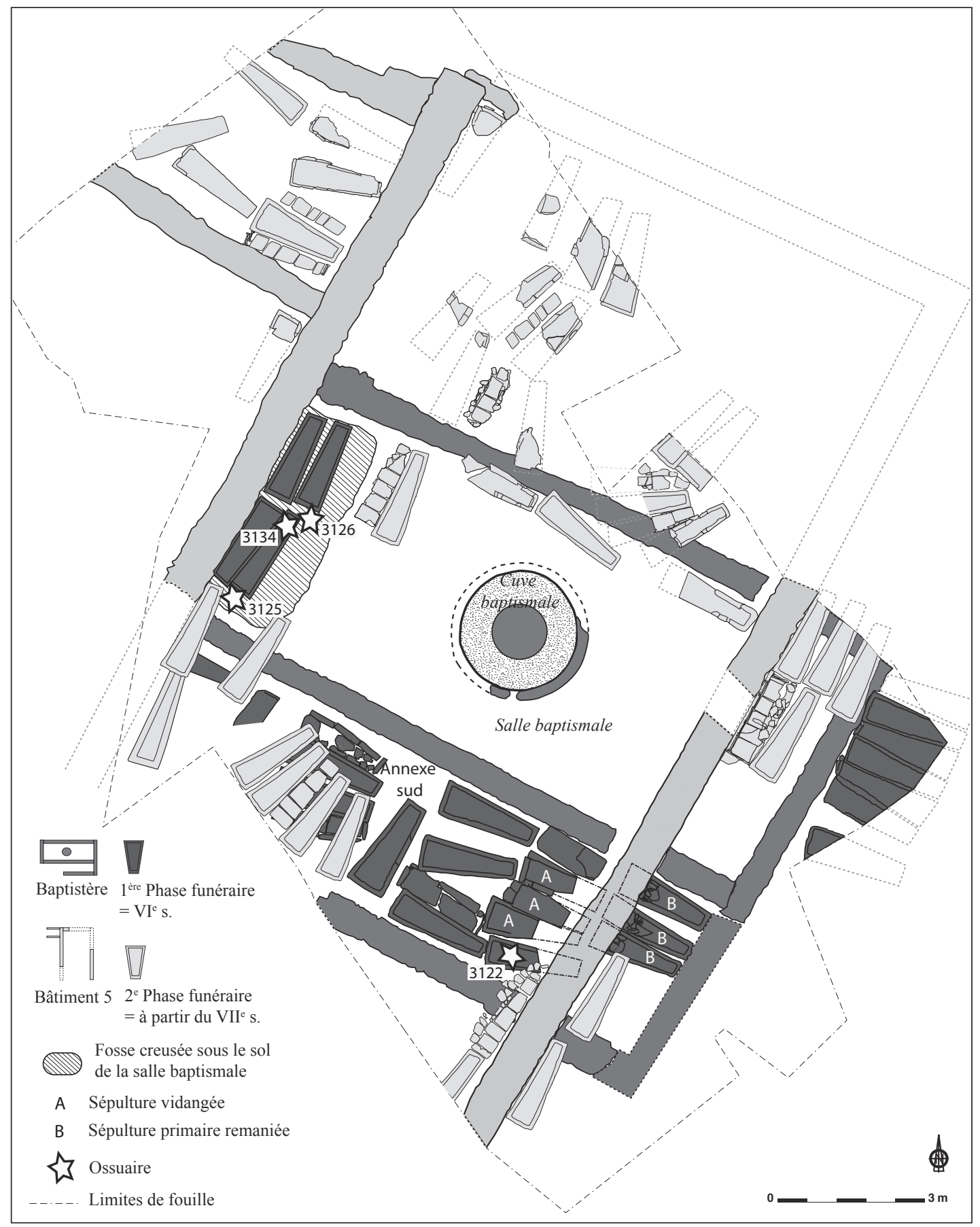

Fig. 1- Occupation funéraire du secteur baptismal du sanctuaire Saint-Julien de Brioude à l'époque mérovingienne.

Fig. 1-Plan of the burial area of the sanctuary of Saint-Julien, Brioude, during the Merovingian period. 
Toutefois, une apparente continuité, à la fois spatiale et typologique, caractérise ces deux phases funéraires. L'élévation du bâtiment 5 sur le baptistère a entraîné diverses perturbations d'éléments architecturaux et funéraires ainsi que la manipulation d'ossements appartenant à la première phase par les occupants de la phase suivante. Nous observons deux principaux gestes :

- dans l'annexe sud du baptistère (fig. 1), trois sarcophages démunis de leur couvercle et partiellement détruits par l'installation du mur est du bâtiment 5, se sont révélés vides, présumant une vidange des squelettes originellement présents ;

- dans le même secteur, trois autres sarcophages (fig. 1), partiellement tronqués par ce même mur, présentent un couvercle intact scellé par du mortier. Les restes osseux décrivent un désordre partiel : les ossements de la partie supérieure de chaque squelette reposent disloqués sur les membres inférieurs en place. Ces sépultures primaires remaniées sont donc la conséquence de l'élévation du mur est du bâtiment 5. Celui-ci venant tronquer l'extrémité céphalique de ces trois tombes, les nouveaux occupants du site ont choisi de repousser les ossements vers le pied des sarcophages, préservant ainsi l'intégrité des squelettes.

En plus de ces tombes vidangées et remaniées, quatre ossuaires ont été mis au jour à l'intérieur du baptistère. Ces ossements ont été assimilés à la première phase funéraire par les archéologues. L'un d'eux (ossuaire 3122), découvert dans l'annexe sud du baptistère, occupe l'un des sarcophages partiellement détruit par le mur est du bâtiment 5 (fig. 1). Sa position accolée aux sarcophages vidangés, nous suggère l'origine de l'ensemble ou d'une partie des ossements ainsi rassemblés. Nous faisons ici l'hypothèse qu'il s'agit de la vidange de sépultures primaires.

À première vue, l'origine des trois autres ossuaires découverts, cette fois-ci, dans la salle baptismale (fig. 1), est plus délicate à appréhender. Or, le mode de constitution de tels dépôts dépend d'un contexte qu'il convient de décrire en vue de reconnaître et interpréter les gestes dont ils dépendent (Blaizot 1997).

Notre objectif ici est de comprendre au mieux le fonctionnement de ces ossuaires découverts dans ce contexte tout à fait singulier qu'est le baptistère. Nous tâcherons donc de déterminer les conditions de leur mise en place avant de décrire plus précisément les caractéristiques ostéologiques propres à chaque dépôt.

\section{MATÉRIEL}

\section{L'origine des ossuaires de la salle baptismale}

Une large fosse a été creusée dans le sol de béton de la salle baptismale afin d'entreposer quatre inhumations primaires en sarcophages pendant la phase de fonctionnement du baptistère (fig. 1). La moitié nord de la fosse est intacte alors que la moitié sud a subi des remaniements contemporains de la construction du bâtiment 5 :

- La moitié nord de la fosse, contient deux sarcophages complets recouverts par un sol de mortier nivelé à l'altitude du terrazzo de la salle baptismale. Ceci prouve la contemporanéité entre la fosse et le baptistère. Une plaquette de marbre insérée dans l'un des couvercles des sarcophages est le support de l'épitaphe de Gunsa, décédée à l'âge de 18 ans ${ }^{4}$ le $1^{\text {er }}$ mars 534 ou 597 sous le règne d'un des deux "Théodebert», maîtres de l'Auvergne (Gauthier 2004).

- Dans la moitié sud de la fosse, les deux sarcophages fragmentés et incomplets témoignent d'une réorganisation spatiale : les cuves ont été déplacées l'une contre l'autre, tête-bêche ; la paroi droite du premier sarcophage étant détruite, la position de cette tombe ainsi accolée à la seconde reconstitue sa paroi manquante. L'ossuaire

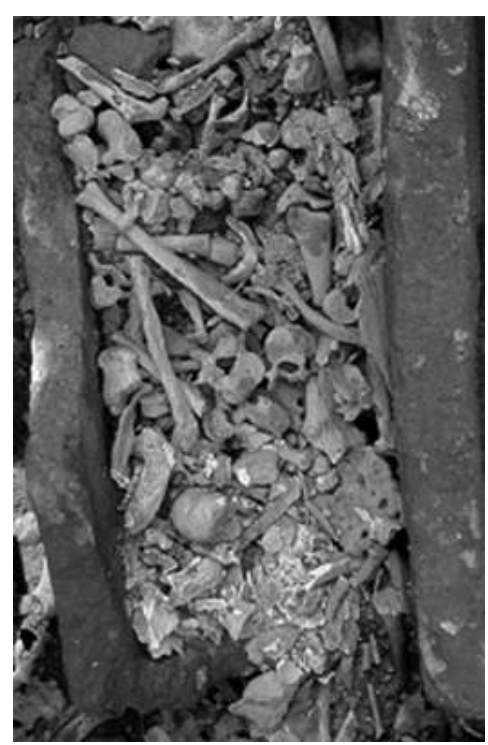

Fig. 2 - Ossuaire 3134.

Fig. 2-Ossuary 3134. 3134 (fig. 2) déposé sur les membres inférieurs de l'individu inhumé dans ce sarcophage et préservé lors de déplacement des cuves, est accolé à ses trois parois ce qui atteste un dépôt postérieur à la réorganisation des cuves. Les deux autres ossuaires (3125 et 3126) sont installés à l'extérieur de cette même tombe, aux deux extrémités.

4. Notre analyse anthropologique du squelette mis au jour dans cette tombe a démontré qu'il s'agissait d'une femme décédée entre 15 et 19 ans, en cohérence avec les données décrites par l'épitaphe. 
L'analyse des données archéologiques et stratigraphiques permet de décrypter les modalités et le contexte de l'aménagement de ces trois ossuaires. Nous constatons d'abord que la réouverture de la fosse, entraînant le remaniement et la destruction partielle des sarcophages, est liée à la reprise du mur ouest du baptistère contigu à cette fosse par le bâtiment 5 (fig. 1). Par ailleurs, si l'on considère les sarcophages de la partie sud de la fosse, on note que la fermeture des cuves et des ossuaires, réalisée par des fragments de couvercles et des blocs de récupération, est approximative, irrégulière et qu'elle se situe dans le niveau de démolition, au-dessus du terrazzo. La réorganisation de la partie sud de la fosse est donc postérieure à l'utilisation de la salle baptismale. Aucune phase d'abandon n'est archéologiquement attestée : la destruction du baptistère et l'élévation du bâtiment 5 se réalisent donc dans un court laps de temps (Gauthier, Thomas à paraître).

Cette analyse tend à nous positionner dans le même contexte que celui décrit pour l'ossuaire 3122 de l'annexe sud: la mise en place des trois ossuaires de la salle baptismale paraît conditionnée par une réorganisation de l'espace directement liée à la construction du bâtiment 5 sur le baptistère. Ces ossements proviennent donc probablement de sépultures primaires vidangées. Contrairement au cas de l'ossuaire 3122, à côté duquel des tombes vides nous laissent très logiquement envisager la provenance des restes osseux, aucune structure inoccupée n'a été découverte à proximité de la salle baptismale. Sans doute faut-il envisager que ces tombes ont été détruites ou qu'elles occupent une zone non fouillée.

L'analyse des restes osseux en position secondaire, confrontée aux données de terrain, révèle une étape charnière entre l'abandon du baptistère et l'élévation du bâtiment 5. Les fondateurs de ce nouvel édifice ont en effet été amenés à manipuler une partie des squelettes de la période précédente : gestes illustrés par le remaniement de sépultures primaires, la vidange de sarcophages et l'aménagement d'ossuaires.

\section{Décompte du nombre minimum d'individus (NMI) représenté par chaque ossuaire}

L'état de conservation des pièces osseuses présentes dans chaque ossuaire est globalement bon. Un faible taux de fragmentation nous a permis l'observation de nombreuses pièces complètes, notamment immatures. Ceci nous a autorisé à évaluer l'état de maturation voire l'âge statural de plusieurs individus immatures à partir de la mesure de certains os longs.

Nous avons procédé au décompte de chaque os ou partie anatomique et avons ainsi établi le NMI de fréquence (Poplin 1976) à partir de la catégorie d'os la mieux représentée. Dans le cas des os pairs, nous avons retenu le meilleur score de l'os latéralisé.

Il est possible d'améliorer ce premier résultat en procédant à des exclusions (NMI affiné). Nous avons tout d'abord tenté de reconstituer les paires d'os appartenant au même individu, afin d'exclure les éléments gauches ou droits qui ne peuvent en aucun cas s'apparier et ainsi augmenter le NMI d'autant d'exclusions (Poplin 1976). La reconnaissance de paires s'est appuyée sur un faisceau de critères morphologiques (taille, robustesse). Une réserve doit toutefois être émise car une marge d'erreur est inévitable dans la recherche de liaisons par symétrie ; leur validité est différente selon les os (Villena Mota et al. 1996). C'est pourquoi, dans les cas où l'appariement nous a seulement paru probable, nous avons considéré ces os comme non exclusifs afin de ne pas surévaluer notre résultat.

Nous avons enfin procédé à un affinement du NMI par classe d'âge en additionnant le meilleur score des catégories d'os adultes à celui des sujets immatures (tabl. I). L'estimation de l'âge des enfants étant possible (Moorrees et al. 1963a, b ; Scheuer, Black 2000), aucun individu représenté ne risque d'être compté deux fois (adulte et immature). Les NMI ont ainsi pu être élevés en toute rigueur.

Notons que le NMI de l'ossuaire 3125 s'élève à 14 bien que le meilleur score d'adultes ne soit que de quatre (réalisé sur les os frontaux, occipitaux, pariétaux, les radius et les os coxaux) tout comme celui des sujets immatures (réalisé sur les os occipitaux et pariétaux droits). En effet, l'ensemble des os temporaux (particulièrement les parties pétreuses) nous a permis d'atteindre un NMI de 14, sans que l'on ait pu les distinguer formellement sur des critères de maturation.

Dès lors si nous envisagions les trois ossuaires de la salle baptismale comme des ensembles distincts, nous pourrions donc considérer qu'un minimum de 32 individus est représenté sur l'ensemble des ossements en position secondaire découverts dans ce secteur. Toutefois rien ne nous permet d'affirmer l'indépendance de chaque structure, ce qui nous interdit d'additionner ainsi ces trois NMI. La suite de notre étude, focalisée sur ces trois ossuaires, démontre en effet qu'il en est tout autrement. 


\begin{tabular}{lccccc}
\hline \hline \multirow{2}{*}{ Localisation ossuaire } & $\mathrm{n}^{\circ}$ & NMI & Immature & Mature & Indéterminé \\
\hline Annexe sud & 3122 & 6 & 1 & 5 & 0 \\
Salle baptismale & 3134 & 8 & 4 & 4 & 0 \\
& 3125 & 14 & 4 & 4 & 6 \\
& 3126 & 10 & 2 & 8 & 0 \\
\hline \hline
\end{tabular}

Tabl. I - Nombre minimum d'individus affiné (NMI) des quatre ossuaires et nombre minimum d'individus de fréquence par classe d'âge.

Table I-Minimum Number of Individuals (MNI) of the four ossuaries based on their frequency by age class.

\section{SÉLECTION ET TRI DES OSSEMENTS}

Les diagrammes ou profils ostéologiques (fig. 3) illustrent la proportion des différentes catégories d'ossements représentées pour chaque ossuaire de la salle baptismale, d'après la méthode mise en place pour les sépultures collectives (Duday 1987). La confrontation des trois profils ostéologiques démontre un résultat sensiblement différent d'un ossuaire à l'autre.

Nous avons déjà observé que les os temporaux donnent le meilleur score de l'ossuaire 3125, avec 14 sujets individualisés (matures et immatures). Ce chiffre semble contradictoire avec la représentation des parties anatomiques infra-crâniennes : si les os de taille petite ou moyenne (os du carpe, du tarse, phalanges, vertèbres et patellas) font complètement défaut, ceux des membres ou des ceintures scapulaire et pelvienne n'atteignent qu'un score maximum de six. En revanche, pour l'ossuaire 3126, ce sont les os longs des membres, notamment les fémurs, qui délivrent le meilleur score (9) avec sept adultes et deux sujets immatures. Comparativement au nombre d'adultes ainsi individualisés, les blocs crânio-faciaux matures sont assez bien représentés (5). Cependant, comme pour l'ossuaire précédent, les os de faibles dimensions sont absents. Enfin, le diagramme de l'ossuaire 3134 nous révèle une assez bonne représentation des vertèbres. De plus, certains os du pied (talus, calcanéus, cuboïdes, métatarses et phalanges) et de la main (hamatums, métacarpes et phalanges) sont présents. Les os longs des membres supérieurs sont mieux représentés que les fémurs et tibias.

Si les trois profils ostéologiques sont différents, nous constatons une compatibilité entre eux : les parties osseuses faisant défaut dans l'un sont présentes dans l'un des deux autres. Par exemple, les os de petites dimensions, quasiment absents dans les ossuaires 3125 et 3126 , sont présents dans l'ossuaire 3134 ; les os longs des membres inférieurs en faible proportion dans les ossuaires 3125 et 3134 sont majoritairement représentés dans l'ossuaire 3126. Nous formulons l'hypothèse que les trois ossuaires de la salle baptismale ont un fonctionnement commun ; il s'agirait de trois dépôts d'ossements appartenant aux mêmes individus.

Afin de tester cette hypothèse, nous avons cherché des «liaisons ostéologiques de deuxième ordre » (Duday 1987, p. 53) dans le but d'étudier la distribution de l'ensemble des vestiges rapportés à un même individu. Ainsi, une liaison ostéologique identifiée entre les restes osseux de deux ossuaires démontrera leur aménagement simultané. Les critères d'identification de liaisons ostéologiques sont divers (collages, appariements, rapports de contiguïté articulaire, identité d'un stade de maturation ou d'un caractère pathologique) et leur validité fut démontrée (Duday 1987). Nous avons évoqué les limites que représente la recherche de liaisons par symétrie (ou appariements) décrites par N. Villena Mota et collaborateurs (1996). De telles réserves sont émises par les auteurs au sujet de la validité des liaisons par contiguïté articulaire. Dès lors, nous avons privilégié les collages et les liaisons talus - calcanéus et os coxalsacrum, démontrées comme les plus performantes.

Nos recherches ont permis d'identifier formellement 12 liaisons de trois natures différentes (fig. 4) : collages (2), contiguïtés articulaires (2) et appariements (8) pour l'ensemble des trois ossuaires. Nous avons exclu six liaisons ostéologiques (une contiguité articulaire et cinq appariements) qui ne nous semblaient que probables.

Les huit appariements retenus ne concernent que les sujets immatures. En fait, 16 ossements pairs, rattachables 


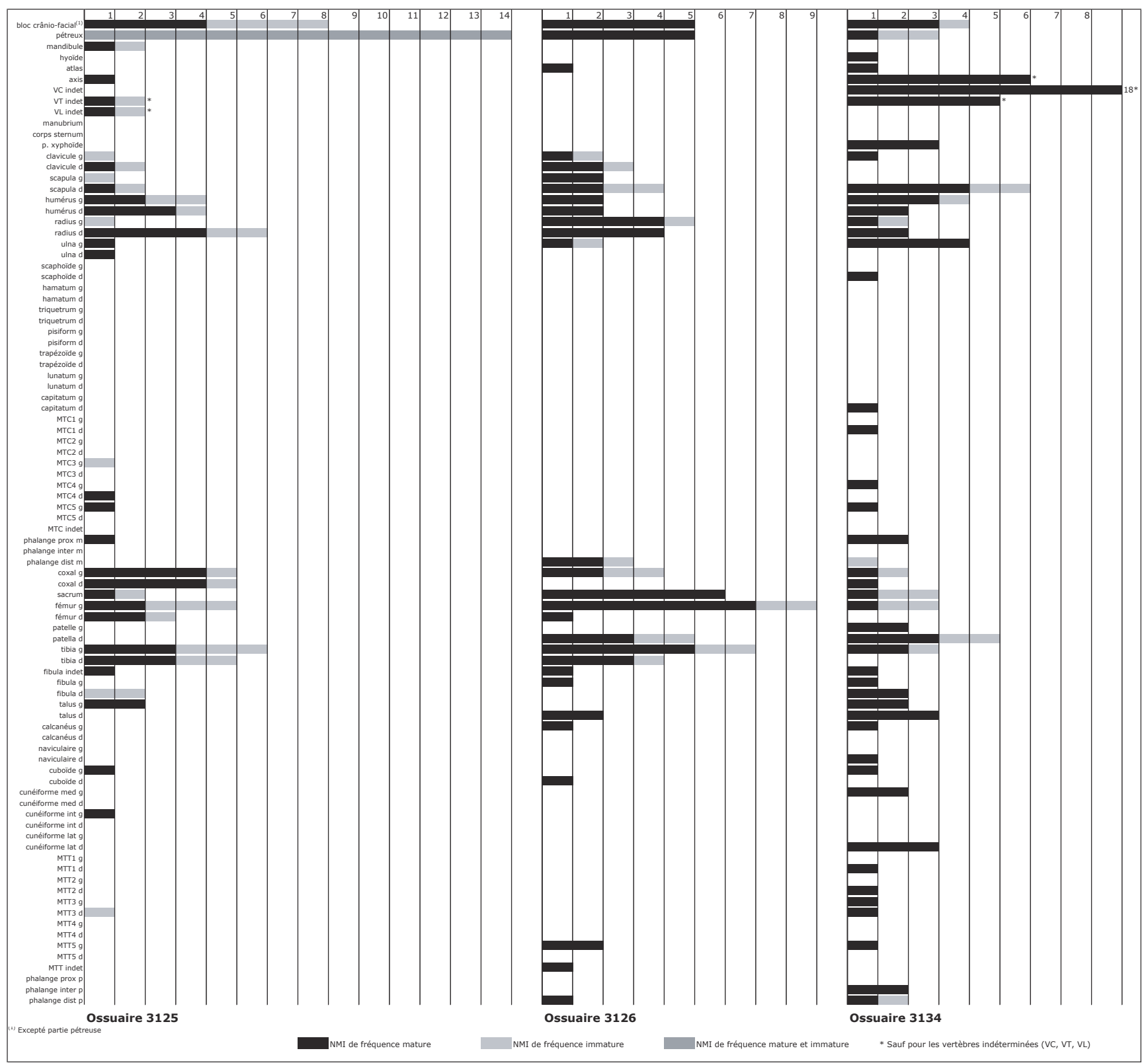

Fig. 3 - Profils ostéologiques des trois ossuaires de la salle baptismale ( $N=$ le nombre des os).

Fig. 3-Bone profiles of the three ossuaries of the baptistery ( $N=$ number of bones).

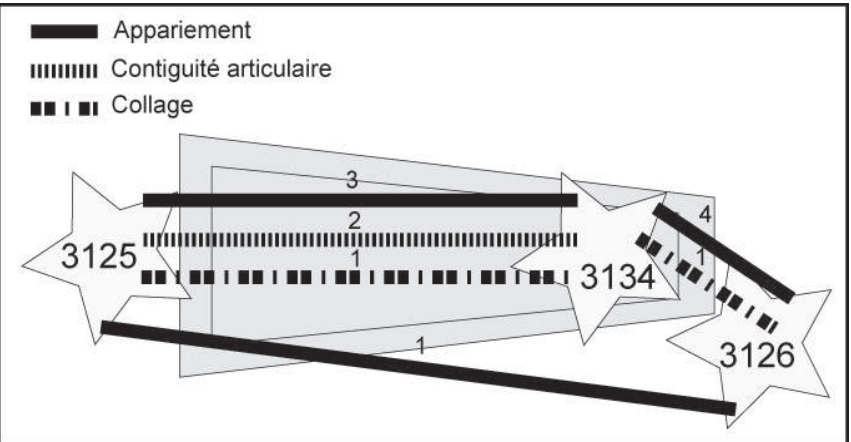

Fig. 4 - Liaisons ostéologiques de second ordre entre les trois ossuaires de la salle baptismale.

Fig. 4-Osteological relations of the second order between the three ossuaries of the baptistery. 
à un minimum de cinq individus immatures, ont été répartis parmi les trois ossuaires (tabl. II). Ce constat ne nous permet néanmoins pas d'affirmer que ce phénomène n'a pas concerné les adultes. D'une part, la reconnaissance d'os pairs est plus aisée sur os immatures, dont les formats montrent une plus grande variabilité, d'autre part, la fragmentation des os matures était plus importante. La fréquente absence des épiphyses des os longs matures a aussi limité leur comparaison.

Ces résultats viennent confirmer l'hypothèse émise : les trois ossuaires de la salle baptismale résultent d'une même action. Si différents dépôts ont été aménagés, les restes osseux des mêmes individus y ont été répartis. Dès lors, nous sommes contraints à réaliser un nouveau décompte des individus, considérant l'ensemble des restes osseux des trois dépôts. Nous obtenons alors un NMI total de 15 avec 10 adultes et 5 immatures représentés. Parmi les adultes, aucune discrimination liée au sexe n'est constatée (au moins trois hommes et une femme sont présents ${ }^{5}$ ) conformément au reste de l'ensemble sépulcral (Gauthier, Thomas à paraître). L'âge des immatures est présenté dans le tableau III.

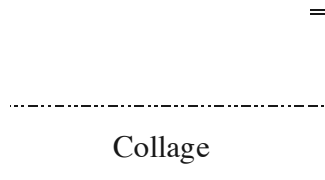

Fémur

Coxal

IM

IM

$\mathrm{X}$

Contiguïté articulaire

Coxal-sacrum

Calcanéus-talus

$\mathrm{AD}$

$\mathrm{AD}$

$\mathrm{X}$

$\mathrm{X}$

Appariement

\begin{tabular}{l} 
Scapula \\
Humérus \\
Radius \\
Coxal \\
Tibia \\
Fémur \\
Fémur \\
Fémur \\
\hline \hline
\end{tabular}

\begin{tabular}{llll} 
IM & $X$ & $X$ & \\
IM & & $X$ & $X$ \\
IM & & $X$ & $X$ \\
IM & & $X$ & $X$ \\
IM & $X$ & & $X$ \\
IM & & $X$ & $X$ \\
IM & $X$ & & $X$ \\
IM & $X$ & & $X$ \\
\hline
\end{tabular}

$\mathrm{X}$

$\mathrm{X}$

$\mathrm{X}$

$\mathrm{X} \quad \mathrm{X}$

$\mathrm{X}$

X

Tabl. II - Catégorie et maturation des ossements concernés par les liaisons ostéologiques de second ordre ( $A D$ : Adulte, IM : Immature, les $X$ indiquent la provenance des os concernés).

Table II-Bone category and maturation concerned by osteological relations of the second order (AD: Adult, IM: Immature, $X$ indicates the origin of bones concerned).

\begin{tabular}{|c|c|c|c|}
\hline & Âge dentaire (1) & Âge statural (2) & Classe \\
\hline Mandibule 1 & $6,1-10,6$ ans & & {$[5-9][10-14]$} \\
\hline Mandibule 2 & $4-7,1$ ans & & {$[1-4][5-9]$} \\
\hline Mandibule 3 & $8-12,5$ ans & & [5-9][10-14] \\
\hline Mandibule 4 & $>12$ ans & & {$[10-14][15-19]$} \\
\hline Fémur* & & $6,5-7$ ans & {$[5-9]$} \\
\hline
\end{tabular}

Tabl. III - Âge des cinq individus immatures présents dans les trois ossuaires de la salle baptismale.

(1) Moorrees et al. 1963a, b. (2) Scheuer, Black 2000.

* La correspondance des âges staturaux de l'ensemble des fémurs aux âges dentaires des quatre mandibules démontre que ce fémur ne peut correspondre à aucune d'entre elles.

Table III-Age of the five immature individuals present in the three ossuaries of the baptistery. (1) Moorrees et al. 1963a, b. (2) Scheuer, Black 2000. * The correspondence between the ages according to stature of all the femurs to the dental ages of the four mandibles shows that this femur does not match any of them.

5. Diagnose sexuelle établie à partir de quatre paires d'os coxaux (Murail et al. 2005). 
Loin d'être aléatoire, l'aménagement des ossuaires de la salle baptismale a été soumis à une action réfléchie visant à sélectionner et trier les ossements en fonction de leur catégorie anatomique. Les crânes ont été préférentiellement déposés à l'extérieur du sarcophage côté tête, les os de petites et moyennes dimensions à l'intérieur du sarcophage, et les os longs des membres inférieurs à l'extérieur du sarcophage côté pieds (fig. 4).

\section{RELATION À L'ÂGE AU DÉCÈS}

L'étude de la composition par âge de l'ensemble de la population inhumée dans ce secteur a permis de mettre en évidence d'autres caractéristiques propres à ces trois ossuaires. Sur l'ensemble de la population inhumée au cours des deux phases, sépultures primaires et ossuaires compris, nous dénombrons 18 sujets immatures ( $<20$ ans) et 72 adultes ( $\geq 20$ ans).

Les sujets immatures ont été répartis en classes d'âge quinquennales, conformément aux tables théoriques de Ledermann (1969). Notre principe directeur est celui de la conformité maximale de nos paramètres paléodémographiques au «schéma de mortalité archaïque » (ou préjennérienne), proposé par P. Sellier (1996). Dans le cas d'alternative (individus à cheval sur deux classes d'âge), nous choisissons systématiquement l'hypothèse qui se conforme le mieux à ce schéma de mortalité archaïque. La distribution des individus immatures révèle que neuf enfants, sur l'ensemble de l'échantillon, sont situés à cheval sur deux classes d'âge. À l'issue de leur répartition définitive, les sujets immatures sont représentés par toutes les classes d'âges, de façon relativement homogène.

La proportion des individus immatures par rapport à celle des adultes peut être discutée à partir du calcul des quotients de mortalité des individus décédés entre 0 et 20 ans (20q0). Considérant l'espace funéraire avec ou sans les ossuaires, les valeurs obtenues (200\%o et $173,91 \%$ ) sont bien en deçà de la fourchette attendue pour une espérance de vie à la naissance de 25 ans $(640 \%)$ ou de 35 ans $(446 \%$ ).

La représentation graphique des quotients et leur comparaison aux quotients théoriques attendus nous mènent à plusieurs constatations. Quels que soient les modes de dépôts observés (sépultures primaires et ossuaires), les courbes révèlent une représentation de la catégorie infantile atypique. Le fait marquant qui nous intéresse ici concerne la classe des 5-9 ans. Ces jeunes sujets inhumés en sépultures individuelles présentent un quotient largement insuffisant par rapport à celui d'une population préindustrielle (fig. 5A). En revanche, ce quotient rentre dans le schéma de mortalité théorique en intégrant les individus issus des ossuaires (fig. 5B).

Ainsi, sur l'ensemble de la période considérée et pour la totalité des individus exhumés, les enfants décédés entre 5 et 9 ans sont présents en proportion normale. Par ailleurs, ces mêmes sujets sont majoritairement représentés dans les ossuaires. Dans la mesure où les ossements de ces ossuaires appartiennent à la première phase funéraire, ce décalage pourrait-il, par là-même, décrire une évolution du recrutement d'une phase chronologique à l'autre ?

La comparaison des profils de mortalité des sujets immatures par phase chronologique (fig. 6) nous permet d'appréhender l'évolution des modalités de recrutement dans cet espace sépulcral, entre le $\mathrm{VI}^{\mathrm{e}} \mathrm{s}$. et la fin de l'époque mérovingienne. La sélection des enfants décédés entre 5 et 9 ans n'a eu lieu qu'à l'époque du fonctionnement du baptistère. Suite à sa destruction, cette classe d'âge ne semble plus avoir eu accès à cette zone d'inhumation. Ce constat soulève plus largement de nombreuses questions quant à la signification de cette stricte inversion du recrutement de cette catégorie d'enfants entre la première et la deuxième phase d'inhumation. Il serait tentant, d'un point de vue contextuel, de mettre en relation l'absence d'inhumation d'enfants décédés entre 5 et 9 ans lors de la phase 2 avec l'abandon de la fonction baptismale. Mais l'augmentation des sépultures d'enfants décédés avant l'âge de 5 ans à cette même période paraît contradictoire avec cette hypothèse. Par ailleurs, contrairement à la phase du baptistère pour laquelle nous disposons d'un espace homogène, cohérent et quasiment complet, la fouille de la deuxième phase funéraire associée au bâtiment 5 n'a pas pu être exhaustive. Par conséquent, rien ne nous permet d'affirmer de façon définitive l'absence réelle de ces sépultures et d'aller plus loin dans l'interprétation de ce biais démographique caractérisant la seconde phase funéraire.

\section{CONCLUSION}

Les deux phases chronologiques qui définissent le secteur baptismal du sanctuaire St-Julien à l'époque mérovingienne se sont succédées dans un court laps de temps. L'élévation du bâtiment 5 sur l'emprise du baptistère a conduit à la vidange de sépultures primaires appartenant à la première phase d'inhumation. Certains 

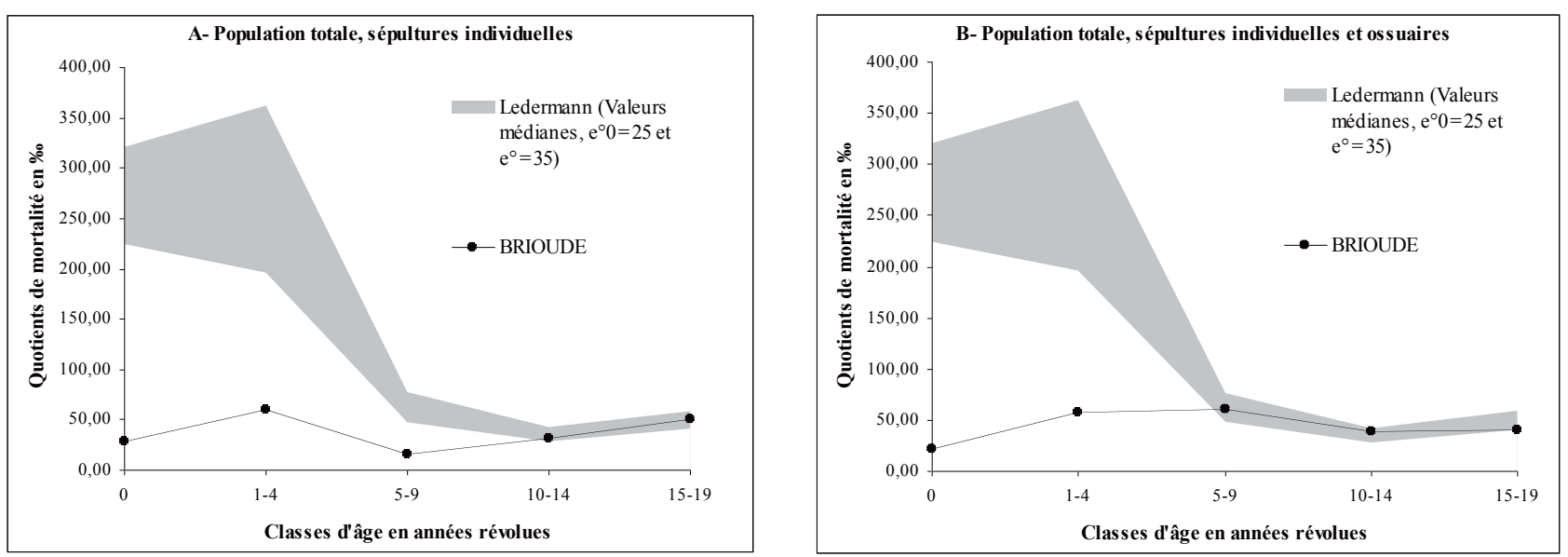

A

Fig. 5 - Comparaison des quotients de mortalité de la population immature avec les tables types de Ledermann (1969) (A-sépultures individuelles et B-sépultures individuelles et ossuaires).

Fig. 5-Comparison of immature mortality quotients with the Ledermann tables (1969) (A-individual burials and B-individual burials and ossuaries).

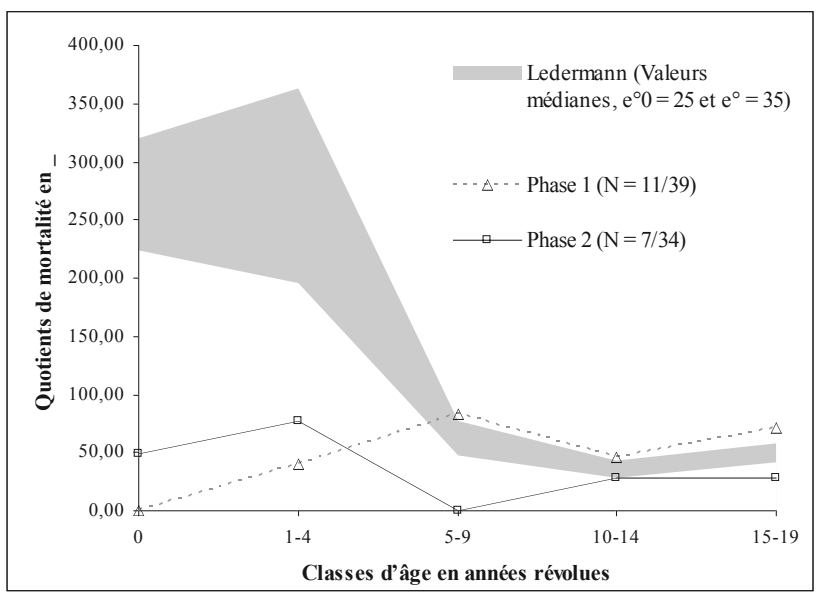

Fig. 6 - Courbes de mortalité de la population immature en fonction des phases chronologiques et comparaison aux standards de Ledermann (1969) ( $N=$ effectif immatureleffectif adulte).

Fig. 6-Immature mortality profiles according to chronological phase compared with the Ledermann tables (1969) ( $N=$ number of immature/ number of adult).

de ces restes osseux, ainsi déplacés par les fondateurs du nouvel édifice, ont été rassemblés en ossuaires.

À l'instar de nombreuses nécropoles historiques, les os humains découverts en position secondaire dans le baptistère de Brioude préfigurent la manifestation d'une contrainte imposée par la surface disponible (Blaizot
1997). En effet, cette manipulation d'ossements ne semble dépendre que de contraintes d'ordre pratique liées au réaménagement de l'espace lors de la construction du bâtiment 5 . Un tel contexte nous engage, en toute logique, à une certaine prudence quant à l'intention «funéraire » du geste à l'origine de ces dépôts, dans la mesure où il ne 
semble pas directement sous-tendu par des rites ou des croyances. Il faut toutefois souligner le caractère organisé de ces dépôts. Les trois ossuaires de la salle baptismale ont en fait recueilli les restes de mêmes individus et résultent d'une action unique. Loin d'être aléatoires, ces amas osseux relèvent d'un geste réfléchi visant à la sélection et au tri des restes squelettiques en fonction de leur catégorie anatomique.

Réintégrée dans l'analyse globale de la population inhumée, l'étude de ces ossuaires participe à la meilleure compréhension de la gestion funéraire de la nécropole. Notre approche de la composition par âge des différentes structures a démontré une forte proportion de la classe des 5-9 ans dans les ossuaires découverts dans le baptistère. Ces individus sont pourtant absents du reste de l'espace d'inhumation, ce qui nous laisse envisager qu'un secteur précis de l'espace sépulcral leur était originellement réservé. Leurs sépultures pourraient avoir été détruites lors de l'élévation du bâtiment 5.
D’une façon générale, l'ensemble de ces données nous informe ici des gestes entretenus par une population vis-à-vis des morts de la génération qui la précède, dans ce contexte singulier qu'est le baptistère de Brioude; le seul actuellement à avoir livré des sépultures dont la contemporanéité avec son fonctionnement est attestée. D'un point de vue archéologique, nous avions souligné l'apparente continuité qui réside entre les deux phases funéraires, tant dans le temps et l'espace que dans le type d'architecture funéraire. Si l'interprétation de ce que sous-tendent les gestes que nous avons ainsi pu mettre en évidence serait mal venue, le caractère non aléatoire de ces manipulations d'ossements nous semble décrire non seulement une continuité entre les deux périodes d'inhumations, mais également une certaine attention tenue à l'égard de la première par la seconde. 


\section{BIBLIOGRAPHIE}

Blaizot (F.) 1997, L'apport des méthodes de la paléoanthropologie funéraire à l'interprétation des os en situation secondaire dans les nécropoles historiques, Archéologie médiévale 26 : 1-22.

Dhenin (M.), Gauthier (F.) 2005, Découverte de monnaies d'argent de Théodebert I (534-548) en fouilles archéologiques à Brioude, Bulletin de la Société Numismatique Française 4 : 66-72.

Duday (H.) 1987, Contribution des observations ostéologiques à la chronologie interne des sépultures collectives, in H. Duday, C. Masset (éds), Anthropologie physique et Archéologie, Méthodes d'étude des sépultures, CNRS, Paris, p. 51-59.

GAUTHIER (F.) 2004, Inscriptions paléochrétiennes découvertes dans le baptistère de Saint-Julien de Brioude (Haute-Loire), Hortus artium medievalium 10 : 211-215.

GAuthier (F.), Thomas (A.) à paraître, Réorganisation spatiale et transferts fonctionnels dans le sanctuaire de Saint-Julien de Brioude au début du VII $\mathrm{s}$. : un nouvel édifice funéraire implanté dans l'emprise du baptistère, in Brioude aux temps carolingiens, Actes du colloque international de Brioude 12-15 septembre 2007, en partenariat avec le CERCOR et l'Almanach de Brioude.

LeDERMANN (S.) 1969, Nouvelles tables-types de mortalité, INED (Travaux et Documents 53), Presses Universitaire de France, Paris.

Moorrees (C.F.A.), Fanning (E.A.), Hunt (E.E.Jr.) 1963a, Age variation of formation stages for ten permanent teeth, Journal of Dental Research 42: 1490-1502.
Moorrees (C.F.A.), Fanning (E.A.), Hunt (E.E.Jr.) 1963b, Formation and resorption of three deciduous teeth in children, American Journal of Physical Anthropology 21: 205-213.

Murail (P.), BrůžEK (J.), HouËT (F.) 2005, DSP: a tool for probabilistic sex diagnosis tool using worldwide variability in hip-bone measurements, Bulletins et Mémoires de la Société d'Anthropologie de Paris, n.s., 17 : 167-176.

Poplin (F.) 1976, Remarques théoriques et pratiques sur les unités utilisées dans les études d'ostéologie quantitative, particulièrement en archéologie préhistorique, in IX $X^{e}$ Congrès de l'UISPP (Nice, 1976, prétirage), Colloque "thèmes spécialisés », CNRS, Nice, p.124-141.

Scheuer (L.), Black (S.) 2000, Developmental Juvenile Osteology, Academic Press, New York, 591 p.

Sellier (P.) 1996, La mise en évidence d'anomalies démographiques et leur interprétation: population, recrutement et pratiques funéraires du tumulus de Courtesoult, in J.-F. Piningre (éd.), Nécropoles et société au premier âge du Fer : le tumulus de Courtesoult (HauteSaône), Éditions de la Maison des Sciences de l'Homme, Paris (DAF 54), p. 188-202.

Villena-Mota (N.), Duday (H.), HouËt (F.) 1996, De la fiabilité des liaisons ostéologiques, Bulletins et Mémoires de la Société d'Anthropologie de Paris, n.s., 8, 3-4 : 373-384. 